\title{
Deep Learning at Scale and at Ease
}

\author{
WEI WANG, National University of Singapore \\ GANG CHEN, Zhejiang University \\ HAIBO CHEN, NetEase Inc. \\ TIEN TUAN ANH DINH, JINYANG GAO, BENG CHIN OOI, KIAN-LEE TAN, \\ and SHENG WANG, National University of Singapore \\ MEIHUI ZHANG, Singapore University of Technology and Design
}

Recently, deep learning techniques have enjoyed success in various multimedia applications, such as image classification and multimodal data analysis. Large deep learning models are developed for learning rich representations of complex data. There are two challenges to overcome before deep learning can be widely adopted in multimedia and other applications. One is usability, namely the implementation of different models and training algorithms must be done by nonexperts without much effort, especially when the model is large and complex. The other is scalability, namely the deep learning system must be able to provision for a huge demand of computing resources for training large models with massive datasets. To address these two challenges, in this article we design a distributed deep learning platform called SINGA, which has an intuitive programming model based on the common layer abstraction of deep learning models. Good scalability is achieved through flexible distributed training architecture and specific optimization techniques. SINGA runs on both GPUs and CPUs, and we show that it outperforms many other state-of-the-art deep learning systems. Our experience with developing and training deep learning models for real-life multimedia applications in SINGA shows that the platform is both usable and scalable.

CCS Concepts: • Computing methodologies $\rightarrow$ Neural networks; $\bullet$ Information systems $\rightarrow$ Multimedia and multimodal retrieval; • Software and its engineering $\rightarrow$ Data flow architectures;

Additional Key Words and Phrases: Multimedia, deep learning, distributed training

ACM Reference Format:

Wei Wang, Gang Chen, Haibo Chen, Tien Tuan Anh Dinh, Jinyang Gao, Beng Chin Ooi, Kian-Lee Tan, Sheng Wang, and Meihui Zhang. 2016. Deep learning at scale and at ease. ACM Trans. Multimedia Comput. Commun. Appl. 12, 4s, Article 69 (November 2016), 25 pages.

DOI: http://dx.doi.org/10.1145/2996464

\section{INTRODUCTION}

In recent years, we have witnessed successful adoptions of deep learning in various multimedia applications, such as image and video classification [Krizhevsky et al. 2012;

This work was supported in part by the National Research Foundation (NRF), Prime Minister's Office, Singapore under its Competitive Research Programme (CRP, award NRF-CRP8-2011-08) and A*STAR project 1321202073. G. Chen's work was supported by National Natural Science Foundation of China grant 61472348. M. Zhang's work was funded under the Energy Innovation Research Programme (EIRP, award NRF2014EWTEIRP002-026), which is administered by the Energy Market Authority. The EIRP is a competitive grant call initiative driven by the Energy Innovation Programme Office and funded by the NRF.

Authors' addresses: W. Wang, T. T. A. Dinh, J. Gao, B. C. Ooi, K.-L. Tan, and S. Wang, COM1, 13 Computing Drive, 117417, Singapore; emails: \{wangwei, dinhtta, jinyang.gao, ooibc, tankl, wangsh\}@comp.nus.edu.sg; G. Chen, Zhejiang University, Hangzhou, 310027, PR China; email: cg@cs.zju.edu.cn; H. Chen, 599 Wangshang Rd, Binjiang, Hangzhou, Zhejiang, China, 310052; email: seaokcs@163.com; M. Zhang, 8 Somapah Road, Singapore, 487372; email: meihui_zhang@sutd.edu.sg.

Permission to make digital or hard copies of all or part of this work for personal or classroom use is granted without fee provided that copies are not made or distributed for profit or commercial advantage and that copies bear this notice and the full citation on the first page. Copyrights for components of this work owned by others than the author(s) must be honored. Abstracting with credit is permitted. To copy otherwise, or republish, to post on servers or to redistribute to lists, requires prior specific permission and/or a fee. Request permissions from permissions@acm.org.

2016 Copyright is held by the owner/author(s). Publication rights licensed to ACM.

ACM 1551-6857/2016/11-ART69 \$15.00

DOI: http://dx.doi.org/10.1145/2996464 
Wu et al. 2014], content-based image retrieval [Wan et al. 2014], music recommendation [Wang and Wang 2014], and multimodal data analysis [Wang et al. 2014; Feng et al. 2014; Zhang et al. 2014]. Deep learning refers to a set of feature learning models that consist of multiple layers. Different layers learn different levels of abstractions (or features) of the raw input data [Le et al. 2012]. It has been regarded as a rebranding of neural networks developed 20 years ago, as it inherits many key neural network techniques and algorithms. However, deep learning exploits the fact that high-level abstractions are better at representing the data than raw, handcrafted features, thus achieving better performance in learning. Its recent resurgence is mainly fueled by higher than ever accuracy obtained in image recognition [Krizhevsky et al. 2012]. Three key factors behind deep learning's remarkable achievement are the advances of neural net structures, immense computing power, and the availability of massive training datasets, which together enable us to train large models to capture the regularities of complex data more efficiently than what was possible 20 years ago.

There are two challenges in bringing deep learning to wide adoption in multimedia applications (and other applications for that matter). The first challenge is usability, namely the implementation of different models and training algorithms must be done by nonexperts with little effort. The user must be able to choose among many existing deep learning models, as different multimedia applications may benefit from different models. For instance, the deep convolutional neural network (DCNN) is suitable for image classification [Krizhevsky et al. 2012], recurrent neural network (RNN) for language modeling [Mikolov et al. 2011], and deep autoencoders for multimodal data analysis [Wang et al. 2014; Feng et al. 2014; Zhang et al. 2014]. Furthermore, the user must not be required to implement most of these models and training algorithms from scratch, as they are too complex and costly. An example of complex models is the GoogleLeNet [Szegedy et al. 2014], which comprises 22 layers of 10 different types. Training algorithms are intricate in details. For instance, the back-propagation (BP) algorithm [LeCun et al. 1996] is notoriously difficult to debug.

The second challenge is scalability, in which the deep learning system must be able to provision for a huge demand of computing resources for training large models with massive datasets. As larger training datasets and bigger models are being used to improve accuracy [Ciresan et al. 2010; Le et al. 2012; Szegedy et al. 2014], the memory requirement for training the model may easily exceed the capacity of a single CPU or GPU. In addition, the computational cost of training may be too high for a single commodity server, which results in unreasonably long training time. For instance, it takes 10 days [Yadan et al. 2013; Paine et al. 2013] to train the DCNN [Krizhevsky et al. 2012] with 1.2 million training images and 60 million parameters using one GPU. ${ }^{1}$

Addressing both usability and scalability challenges requires a distributed training platform that supports various deep learning models, comes with an intuitive programming model, and is scalable. Popular deep learning systems, including Caffe [Jia et al. 2014], Torch [Collobert et al. 2011], and Theano [Bastien et al. 2012], address the first challenge but fall short at the second challenge (they are not designed for distributed training). Several systems support distributed training [Paine et al. 2013; Yadan et al. 2013; Krizhevsky 2014], but they are model specific and do not generalize well to other models. General distributed platforms such as MapReduce and epiC [Jiang et al. 2014] achieve good scalability, but they are designed for general data processing. As a result, they lack both the programming model and system optimization specific to deep learning, hindering the overall usability and scalability. There are several specialized distributed platforms [Dean et al. 2012; Coates et al. 2013; Chilimbi et al. 2014] that exploit deep learning-specific optimization and hence are able to achieve high training

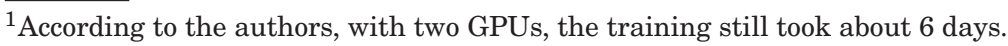


throughput. However, they forgo usability issues: the platforms are closed source and no details of their programming models are given, rendering them unusable by multimedia users.

In this article, we present our effort in bringing deep learning to the masses. In particular, we extend our previous work [Ooi et al. 2015; Wang et al. 2015a] on distributed training of deep learning models. In Wang et al. [2015a], we designed and implemented an open-source distributed deep learning platform, called SINGA, ${ }^{2}$ which tackles both usability and scalability challenges at the same time. In this work, we introduce optimization techniques and GPU support for SINGA. SINGA provides a simple, intuitive programming model that makes it accessible even to nonexperts. SINGA's simplicity is driven by the observation that both the structures and training algorithms of deep learning models can be expressed using a simple abstraction: the neuron layer (or layer). In SINGA, the user defines and connects layers to form the neural network model, and the runtime transparently manages other issues pertaining to the distributed training, such as partitioning, synchronization, and communication. Particularly, the neural network is represented as a dataflow computation graph with each layer being a node. During distributed training, the graph is partitioned and each subgraph can be trained on CPUs or GPUs. SINGA's scalability comes from its flexible system architecture and specific optimization. Both synchronous and asynchronous training frameworks are supported with a range of built-in partitioning strategies, which enables users to readily explore and find an optimal training configuration. Optimization techniques, including minimizing data transferring and overlapping computation and communication, are implemented to reduce the communication overhead from distributed training.

In summary, this article makes the following contributions:

(1) We present SINGA, a distributed platform that is designed to train deep learning models for multimedia and other applications. SINGA offers a simple and intuitive programming model based on the layer abstraction.

(2) We describe SINGA's distributed architecture and optimization for reducing the communication overhead in distributed training.

(3) We demonstrate SINGA's usability by describing the implementation of three multimedia applications: multimodal retrieval, dimensionality reduction, and sequence modeling.

(4) We evaluate SINGA's performance by comparing it to other open-source systems. The results show that SINGA is scalable and outperforms other systems in terms of training time.

This article is an extension of our conference paper [Wang et al. 2015a]. In Wang et al. [2015a], we presented the basic SINGA framework for a homogeneous architecture (where we consider only CPU nodes). In this work, we extend the framework to a heterogeneous setting that consists of both GPU and CPU processors. Optimization techniques in terms of reducing communication overhead from distributed training are introduced here. Correspondingly, we conducted experiments on GPUs in comparison with existing systems. The rest of the article is organized as follows. Section 2 provides the background on training deep learning models and related work. An overview of SINGA as a platform follows in Section 3. The programming model is discussed in Section 4. We discuss SINGA architecture and training optimization in Section 5. The experimental study is presented in Section 6, and we conclude in Section 7.

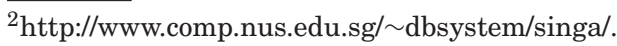




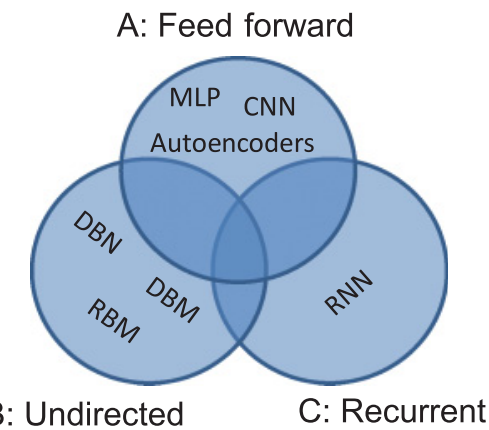

Fig. 1. Deep learning model categorization.

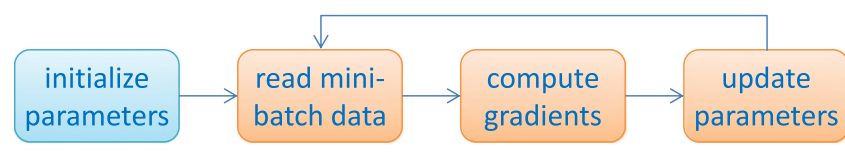

Fig. 2. Flow of the SGD algorithm.

\section{BACKGROUND}

Deep learning is considered as a feature learning technique. A deep learning model typically consists of multiple layers, each associated with a feature transformation function. After going through all layers, raw input features (e.g., pixels of images) are converted into high-level features that are used for the task of interest (e.g., image classification).

\subsection{Models and Training Algorithms}

We group popular deep learning models into three categories based on the connection types between layers, as shown in Figure 1. Category A consists of feed-forward models, wherein the layers are directly connected. The extracted features at higher layers are fed into prediction or classification tasks (e.g., image classification) [Krizhevsky et al. 2012]. Example models in this category include multilayer perceptron (MLP), convolutional neural networks (CNNs), and autoencoders. Category $B$ contains models whose layer connections are undirected. These models are often used to pretrain other models [Hinton and Salakhutdinov 2006] (e.g., feed-forward models). The deep belief network (DBN), deep Boltzmann machine (DBM), and restricted Boltzmann machine (RBM) are examples of such models. Category $C$ comprises RNNs, which are models that have recurrent connections. They are widely used for modeling sequential data in which prediction of the next position is affected by previous positions. Language modeling [Mikolov et al. 2011] is a popular application of RNNs.

A deep learning model has to be trained to find the optimal parameters for the transformation functions. The training quality is measured by a loss function (e.g., cross-entropy loss) for each specific task. Since the loss functions are usually nonlinear and nonconvex, it is difficult to get closed-form solutions. A common approach is to use the stochastic gradient descent (SGD) algorithm shown in Figure 2. SGD initializes the parameters with random values and then iteratively refines them to reduce the loss based on the computed gradients. There are three typical algorithms for gradient computation corresponding to the preceding three model categories: BP, contrastive divergence (CD), and back-propagation through time (BPTT). 
Table I. Comparison of Existing Open-Source Systems (and Libraries)

\begin{tabular}{|l|l|l|l|l|l|l|l|}
\hline & SINGA & Torch & Caffe & MxNet & TensorFlow & Theano & CNTK \\
\hline Programming Style & I & I & I & I, D & D & D & D \\
\hline Flexibility and Extensibility & $* * *$ & $* * * *$ & $* *$ & $* * * *$ & $* * * *$ & $* * * *$ & $* * * *$ \\
\hline Distributed Training & $* * * *$ & $* *$ & $* *$ & $* * *$ & $* * *$ & $* *$ & $* * *$ \\
\hline Hardware Support & $* *$ & $* * *$ & $* * *$ & $* *$ & $* * *$ & $* * *$ & $* *$ \\
\hline
\end{tabular}

\subsection{Related Work}

Due to its outstanding capabilities in capturing complex regularities of multimedia data (e.g., image and video), deep learning techniques are being adopted by more and more multimedia applications, such as image retrieval [Wan et al. 2014], multimodal retrieval [Wang et al. 2014, 2015b], and sentiment analysis [You et al. 2015]. In recent years, we have witnessed fast increase of deep learning models' depth, from tens of layers (e.g., AlexNet [Krizhevsky et al. 2012] and VGG [Simonyan and Zisserman 2014]) to hundreds of layers [He et al. 2015]. It has been shown that deeper models work better for the ImageNet challenge task [Szegedy et al. 2014; Simonyan and Zisserman 2014]. Meanwhile, training datasets are also becoming larger, from 60,000 images in the MNIST and Cifar datasets to millions of images in the ImageNet dataset. Complex deep models and massive training datasets require a huge amount of computing resources for training.

Different applications use different deep learning models. It is essential to provide a general deep learning system for nonexperts to implement their models without much effort. Some distributed training approaches have been proposed [Paine et al. 2013; Yadan et al. 2013; Krizhevsky et al. 2012]. They are specifically optimized for training the AlexNet model [Krizhevsky et al. 2012] and thus cannot generalize well to other models. Other general distributed deep learning platforms [Dean et al. 2012; Coates et al. 2013; Chilimbi et al. 2014] exploit deep learning-specific optimization and hence are able to achieve high training throughput. However, they are closed source and there are no details of the programming model, rendering them unusable to developers. There are also some popular open-source systems for training deep learning models on a single node, including TensorFlow [Abadi et al. 2015], Caffe [Jia et al. 2014], Torch [Collobert et al. 2011], MxNet [Chen et al. 2015], Theano [Bastien et al. 2012] and CNTK [Agarwal et al. 2014]. Distributed training is being added for some of these systems. We briefly compare these systems (or libraries) in Table I, which mainly includes the usability (the first two rows) and efficiency (the last two rows).

Two major programming styles are used in these systems: imperative programming and declarative programming. SINGA, Caffe, and Torch use imperative programming (denoted as I), which is easy to get started and debug. TensorFlow, Theano, and CNTK follow the declarative programming model (denoted as D), where users simply declare the learning objective and the system creates a computation graph (dataflow graph) for automatically optimizing the learning objective. The computation graph provides opportunities for speed and memory optimization [Chen et al. 2016b] but is not easy to debug and requires some effort to get started.

Layer is an inherent abstraction of neural networks. Almost all systems provide the layer abstraction (they may use different names). Caffe uses Layer as the lowest computation unit. Other systems provide Tensor abstractions for algebra operations. Caffe's layer abstraction was designed for feed-forward neural networks and was latter extended to support RNN but has no support for energy models. SINGA's layer abstraction supports all three popular neural networks. Other systems, since they provide both Tensor and Layer abstractions, are more flexible to implement in general machine learning algorithms. 


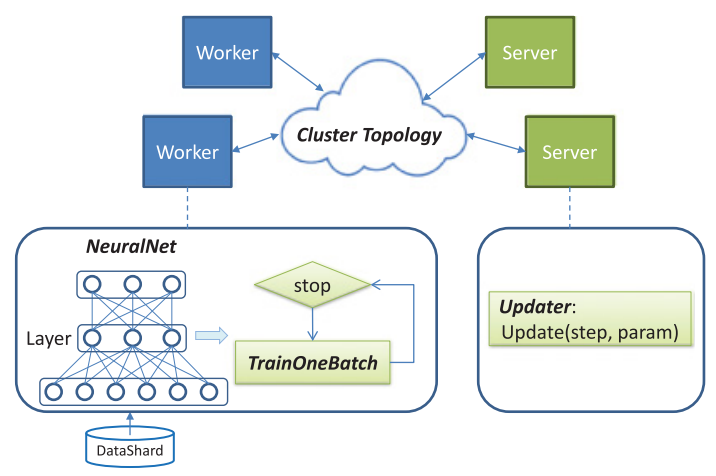

Fig. 3. SINGA overview.

Distributed training is becoming more and more important. All systems in the table support distributed training to improve training efficiency. Caffe, Torch, and Theano support training with multi-GPU cards on a single node. However, training in a GPU cluster is not officially supported. SINGA, TensorFlow, CNTK, and MxNet are designed with distributed training considered. SINGA is flexible to provide distributed training frameworks (Section 5.2) and exploits asynchronous data transferring and hybrid partitioning to optimize the communication cost (Section 5.4). MxNet has a fixed two-layer distributed training architecture. TensorFlow and CNTK are also working on improving distributed training. For example, CNTK uses parameter compressing to reduce communication cost [Seide et al. 2014], and TensorFlow uses synchronous SGD with auxiliary workers [Chen et al. 2016a].

Hardware acceleration is vital for the success of deep learning models. All systems in the table use CUDA and the cuDNN library ${ }^{3}$ to run computation-intensive operations on GPUs, such as convolution and pooling. Caffe, Torch, and Theano have the partial support of OpenCL for deployment on small devices.

\section{OVERVIEW}

SINGA trains deep learning models using SGD over the worker-server architecture, as shown in Figure 3. Workers compute parameter gradients, and servers perform parameter updates. To start a training job, the user (or programmer) submits a job configuration specifying the following four components:

-A NeuralNet describing the neural network (or neural net) structure with the detailed layers and their connections. SINGA comes with many built-in layers (Section 4.1.2), and users can also implement their own layers for feature transforming or data reading (writing).

-A TrainOneBatch algorithm for training the model. SINGA implements different algorithms (Section 4.1.3) for all three model categories.

-An Updater defining the protocol for updating parameters at the servers (Section 4.1.4).

-A Cluster Topology specifying the distributed architecture of workers and servers. SINGA's architecture is flexible and can support both synchronous and asynchronous training (Section 5).

Given a job configuration, SINGA distributes the training tasks over the cluster and coordinates the training. In each iteration, every worker calls the TrainOneBatch

\footnotetext{
$\overline{{ }^{3} \mathrm{https}: / / d e v e l o p e r . n v i d i a . c o m / c u d n n . ~}$
} 


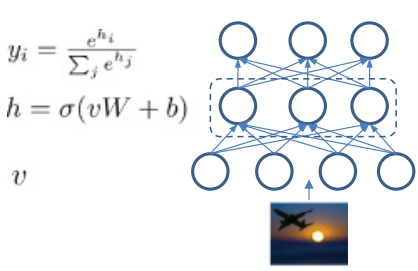

(a) Sample MLP

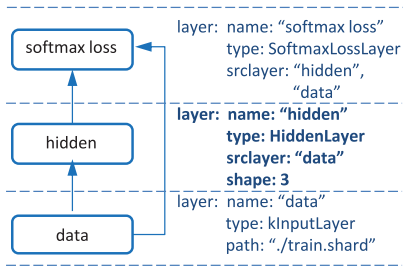

(b) Net configuration

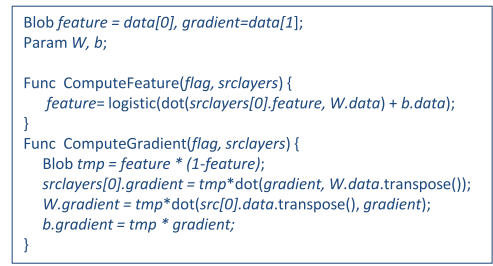

(c) Pseudocode for the hidden layer

Fig. 4. Running example using an MLP.

function to compute parameter gradients. TrainOneBatch takes a NeuralNet object representing the neural net, and it visits (part of) the model layers in an order specific to the model category. The computed gradients are sent to the corresponding servers for updating. Workers then fetch the updated parameters at the next iteration.

\section{PROGRAMMING MODEL}

This section describes SINGA's programming model, particularly the main components of a SINGA job. We use the MLP model for image classification (Figure 4(a)) as a running example. The model consists of an input layer, a hidden feature transformation layer, and a softmax output layer.

\subsection{Programming Abstractions}

4.1.1. NeuralNet. NeuralNet represents a neural net instance in SINGA. It comprises a set of unidirectionally connected layers. Properties and connections of layers are specified by users. The NeuralNet object is passed as an argument to the TrainOneBatch function.

Layer connections in NeuralNet are not designed explicitly; instead, each layer records its own source layers as specified by users (Figure 4(b)). Although different model categories have different types of layer connections, they can be unified using directed edges as follows. For feed-forward models, nothing needs to be done, as their connections are already directed. For undirected models, users need to replace each edge with two directed edges, as shown later in Figure 7. For recurrent models, users can unroll a recurrent layer into directed-connecting sublayers, as shown later in Figure 8.

4.1.2. Layer. Layer is a core abstraction in SINGA. Different layer implementations perform different feature transformations to extract high-level features. In every SGD iteration, all layers in the NeuralNet are visited by the TrainOneBatch function during the process of computing parameter gradients. From the dataflow perspective, we can regard the neural net as a graph where each layer is a node. The training procedure passes data along the connections of layers and invokes functions of layers. Distributed training can be easily conducted by assigning subgraphs to workers.

Figure 5 shows the definition of a base layer. The data field records data (blob) associated with a layer. Some layers may require parameters (e.g., a weight matrix) for their feature transformation functions. In this case, these parameters are represented by Param objects, each with a data field for the parameter values and a gradient field for the gradients. The ComputeFeature function evaluates the feature blob by transforming features from the source layers. The ComputeGradient function computes the gradients associated with this layer. These two functions are invoked by the TrainOneBatch function during training (Section 4.1.3). 


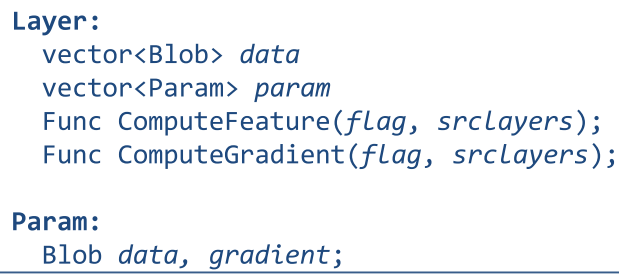

Fig. 5. Layer abstraction.

Table II. Layer Categories

\begin{tabular}{|l|l|}
\hline Category & \multicolumn{1}{|c|}{ Description } \\
\hline Input layers & Load records from file, database, or HDFS. \\
\hline Output layers & Dump records to file, database, or HDFS. \\
\hline Neuron layers & Feature transformation (e.g., convolution). \\
\hline Loss layers & Compute objective loss (e.g., cross-entropy loss). \\
\hline Connection layers & Connect layers when neural net is partitioned. \\
\hline
\end{tabular}

SINGA provides a variety of built-in layers to help users build their models. Table II lists the layer categories in SINGA. For example, the data layer loads a mini-batch of records via the ComputeFeature function in each iteration. Users can also define their own layers for their specific requirements. Figure 4(c) shows an example of implementing the hidden layer $h$ in the MLP. In this example, beside feature blobs there are gradient blobs storing the gradients of the loss with respect to the feature blobs. There are two Param objects: the weight matrix $W$ and the bias vector $b$. The ComputeFeature function rotates (multiply $W$ ), shifts (plus $b$ ) the input features, and then applies nonlinear (logistic) transformations. The ComputeGradient function computes the layer's parameter gradients, as well as the source layer's gradients that will be used for evaluating the source layer's parameter gradients.

4.1.3. TrainOneBatch. The TrainOneBatch function determines the sequence of invoking ComputeFeature and ComputeGradient functions in all layers during each SGD iteration. SINGA implements two TrainOneBatch algorithms for the three model categories. For feed-forward and recurrent models, the BP algorithm is provided. For undirected modes (e.g., RBM), the CD algorithm is provided. Users simply select the corresponding algorithm in the job configuration. Should there be specific requirements for the training workflow, users can define their own TrainOneBatch function following a template shown in Algorithm 1. Algorithm 1 implements the BP algorithm, which takes a NeuralNet object as input. The first loop visits each layer and computes their features, and the second loop visits each layer in the reverse order and computes parameter gradients.

4.1.4. Updater. Once the parameter gradients are computed, workers send these values to servers to update the parameters. SINGA implements several parameter updating protocols, such as AdaGrad [Duchi et al. 2011]. Users can also define their own updating protocols by overriding the Update function.

\subsection{Multimedia Applications}

This section demonstrates the use of SINGA for multimedia applications. We discuss the training of three deep learning models for three different applications: a multimodal deep neural network (MDNN) for multimodal retrieval, an RBM for dimensionality reduction, and an RNN for sequence modeling. 


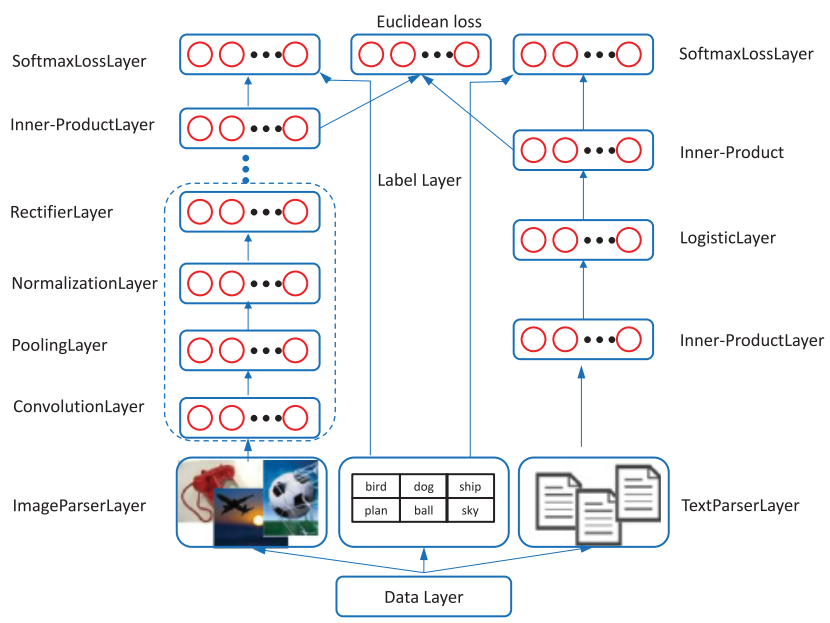

Fig. 6. Structure of an MDNN.

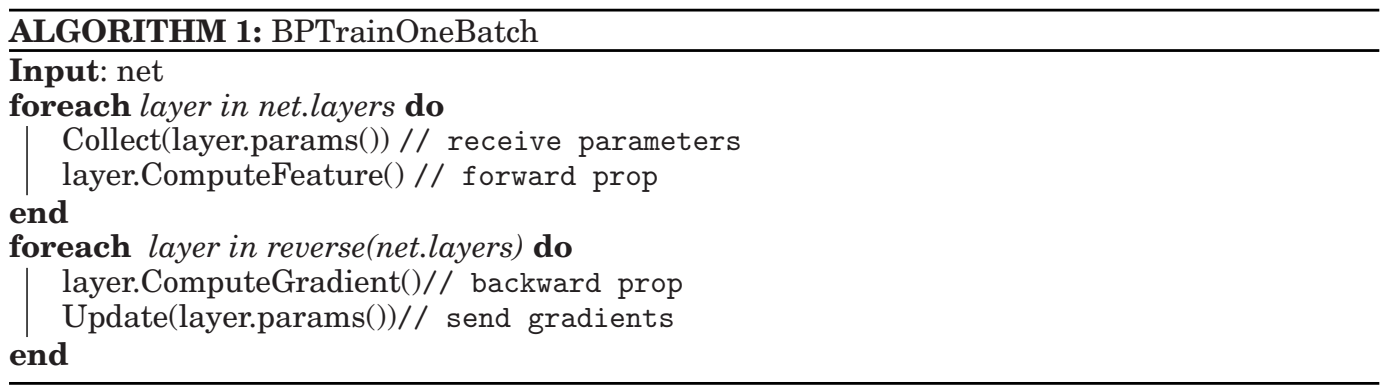

4.2.1. MDNN for Multimodal Retrieval. Feed-forward models such as CNN and MLP are widely used to learn high-level features in multimedia applications, especially for image classification [Krizhevsky et al. 2012]. Here we demonstrate the training of the MDNN [Wang et al. 2015a] using SINGA to extract features for the multimodal retrieval task [Wang et al. 2014; Feng et al. 2014; Shen et al. 2000] that searches objects from different modalities. In MDNN, there is a CNN [Krizhevsky et al. 2012] for extracting image features and an MLP for extracting text features. The training objective is to minimize a weighted sum of (1) the error of predicting the labels of image and text documents using extracted features and (2) the distance between features of relevant image and text objects.

Figure 6 depicts the neural net of the MDNN model in SINGA. We can see that there are two parallel paths: one for text modality and the other for image modality. The data layer reads in records of semantically relevant image-text pairs. The image layer, text layer, and label layer then parse the visual feature, text feature (e.g., tags of the image), and labels, respectively, from the records. The image path consists of layers from DCNN [Krizhevsky et al. 2012], such as the convolutional layer and pooling layer. The text path includes an inner-product (or fully connected) layer, a logistic layer, and a loss layer. The Euclidean loss layer measures the distance of the feature vectors extracted from these two paths. All except the parser layers, which are application specific, are SINGA's built-in layers. Since this model is a feed-forward model, the BP algorithm is selected for the TrainOneBatch function. 


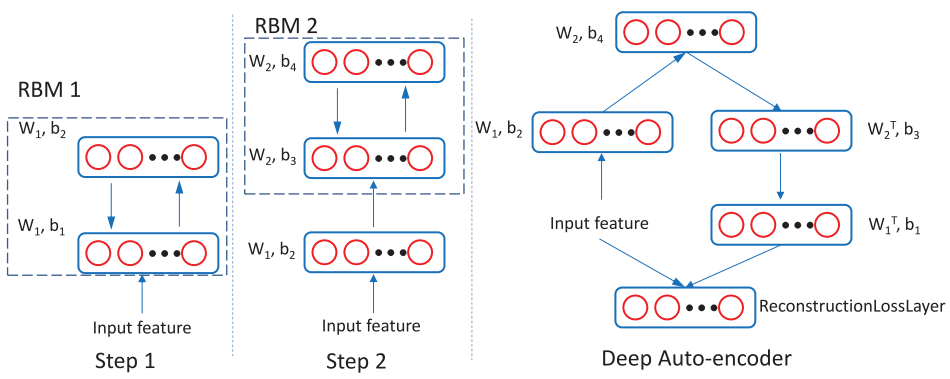

Fig. 7. Structure of RBM and deep autoencoders.

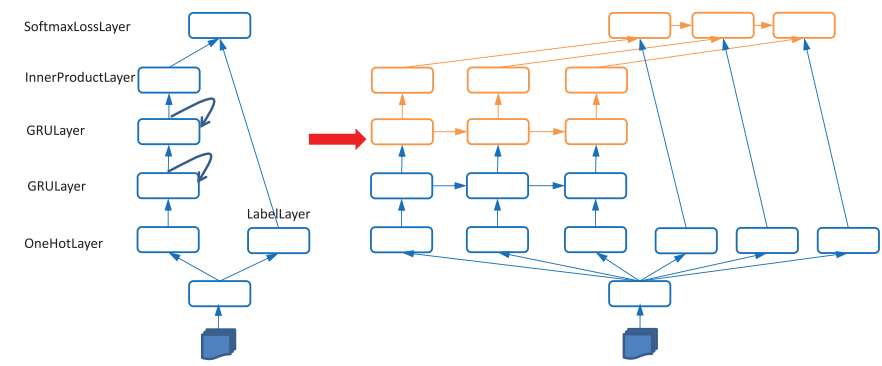

Fig. 8. Structure of a two-stacked Char-RNN before unrolling (left) and after unrolling (right).

4.2.2. RBM for Dimensionality Reduction. RBM is often employed to pretrain parameters for other models. In this example application, we use RBM to pretrain deep autoencoders [Hinton and Salakhutdinov 2006] for dimensionality reduction. Multimedia applications typically operate with high-dimensional feature vectors, which demands large computing resources. Dimensionality reduction techniques, such as principal component Analysis (PCA), are commonly applied in the preprocessing step. Deep autoencoders are reported [Hinton and Salakhutdinov 2006] to have better performance than PCA.

Generally, the deep autoencoders are trained to reconstruct the input feature using the feature of the top layer. Hinton and Salakhutdinov [2006] used RBM to pretrain the parameters for each layer and fine tuned them to minimize the reconstruction error. Figure 7 shows the model structure (with parser layer and data layer omitted) in SINGA. The parameters trained from the first RBM (RBM 1) in step 1 are ported (through checkpoint) into step 2, wherein the extracted features are used to train the next model (RBM 2). Once pretraining is finished, the deep autoencoders are unfolded for fine tuning. SINGA applies the CD algorithm for training RBM and the BP algorithm for fine tuning the deep autoencoder.

4.2.3. RNN for Sequence Modeling. RNNs are widely used for modeling sequential data (e.g., natural language sentences). We use SINGA to train a Char-RNN model ${ }^{4}$ over Linux kernel source code, with each character as an input unit. The model predicts the next character given the current character.

Figure 8 illustrates the net structure of the Char-RNN model. In each iteration, the input layer reads unroll len +1 (unroll len is specified by users) successive characters, (e.g., "int a;") and passes the first unroll_len characters to OneHotLayers (one per layer),

\footnotetext{
${ }^{4}$ https://github.com/karpathy/char-rnn.
} 


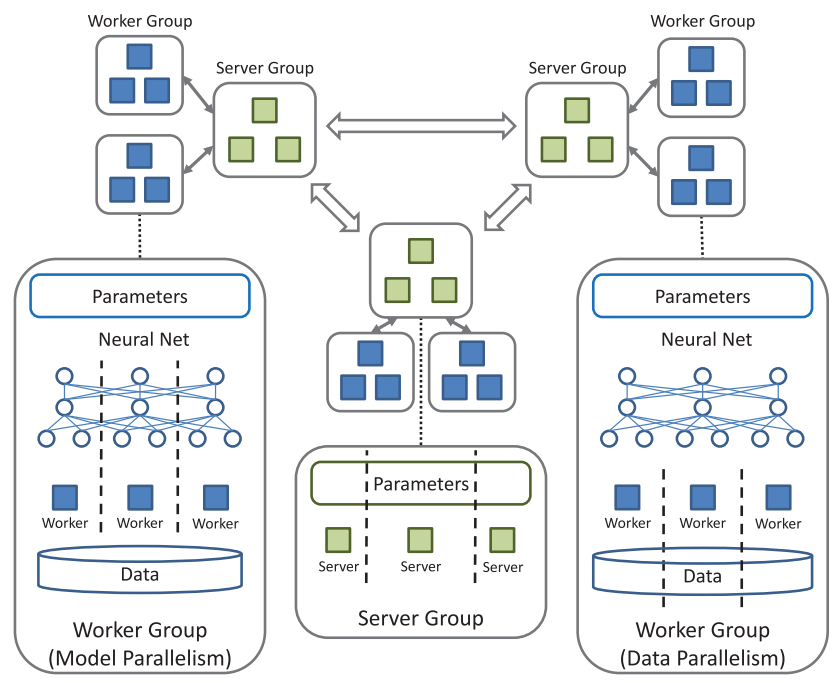

Fig. 9. Logical architecture of SINGA.

and passes the last unroll len characters as labels to the label layer (the label of the $i^{\text {th }}$ character is the $(i+1)^{t h}$ character, i.e., the objective is to predict the next character). The model is configured similarly as for feed-forward models except the training algorithm is BPTT, and unrolling length and connection types are specified for recurrent layers. Different colors are used for illustrating the neural net partitioning, which will be discussed in Section 5.3.

\section{DISTRIBUTED TRAINING}

In this section, we introduce SINGA's architecture and discuss how it supports a variety of distributed training frameworks.

\subsection{System Architecture}

Figure 9 shows the logical architecture, which consists of multiple server groups and worker groups, and each worker group communicates with only one server group. Each server group maintains a complete replica of the model parameters and is responsible for handling requests (e.g., get or update parameters) from worker groups. Neighboring server groups synchronize their parameters periodically. Typically, a server group contains several servers, and each server manages a partition of the model parameters. Each worker group trains a complete model replica against a partition of the training dataset (i.e., data parallelism) and is responsible for computing parameter gradients. All worker groups run and communicate with the corresponding server groups asynchronously. However, inside each worker group, the workers compute parameter updates synchronously for the model replica. There are two strategies to distribute the training workload among workers within a group: by model or by data. More specifically, each worker can compute a subset of parameters against all data partitioned to the group (i.e., model parallelism) or all parameters against a subset of data (i.e., data parallelism). SINGA also supports hybrid parallelism (Section 5.3).

In SINGA, servers and workers are execution units running in separate threads. If GPU devices are available, SINGA automatically assigns $g$ GPU devices ( $g$ is user specified) to the first $g$ workers on each node. A GPU worker executes the layer functions on the GPU if they are implemented using GPU API (e.g., CUDA). Otherwise, the layer 


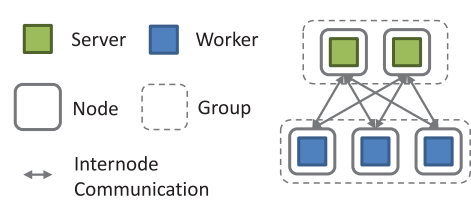

(a) Sandblaster

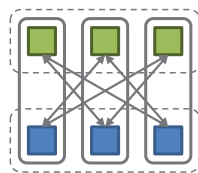

(b) AllReduce

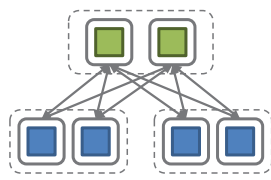

(c) Downpour

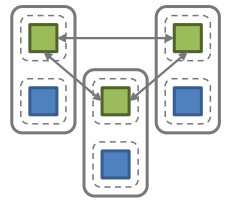

(d) Distributed Hogwild

Fig. 10. Training frameworks in SINGA.

functions execute on the CPU. SINGA provides several linear algebra functions for users to implement their own layer functions. These linear algebra functions have both GPU and CPU implementation, and they determine the running device of the calling thread automatically. In this way, we keep the implementation transparent to users. Workers and servers communicate through message passing. Every process runs the main thread as a stub that aggregates local messages and forwards them to corresponding (remote) receivers. SINGA uses the ZeroMQ library for message passing over the network.

\subsection{Training Frameworks}

In SINGA, worker groups run asynchronously and workers within one group run synchronously. Users can leverage this general design to run both synchronous and asynchronous training frameworks. Specifically, users control the training framework by configuring the cluster topology (i.e., the number of worker (respectively, server) groups and worker (respectively, server) group size. In the following, we will discuss how to realize popular distributed training frameworks in SINGA, including Sandblaster and Downpour from Google's DistBelief system [Dean et al. 2012], AllReduce from Baidu's Deep Image system [Wu et al. 2015], and distributed Hogwild from Caffe [Jia et al. 2014].

5.2.1. Synchronous Training. A synchronous framework is realized by configuring the cluster topology with only one worker group and one server group. The training convergence rate is the same as that on a single node. Figure 10(a) shows the Sandblaster framework implemented in SINGA. A single server group is configured to handle requests from workers. A worker operates on its partition of the model and only communicates with servers handling the related parameters. This framework is typically used if high-performance dedicated servers with large network bandwidth are available [Chilimbi et al. 2014]. Figure 10(b) shows the AllReduce framework in SINGA, in which we bind each worker with a server on the same node so that each node is responsible for maintaining a partition of parameters and collecting updates from all other nodes. This framework is suitable for the single-node multi-GPU case or small GPU clusters. For large clusters, the all-to-all connection would incur a huge amount of communication cost.

Synchronous training is typically limited to a small- or medium-size cluster (e.g. fewer than 100 nodes). When the cluster size is large, the synchronization delay is likely to be larger than the computation time. Consequently, the training cannot scale well.

5.2.2. Asynchronous Training. An asynchronous framework is implemented by configuring the cluster topology with more than one worker group. The training convergence is likely to be different from single-node training, because multiple worker groups are working on different versions of the parameters [Zhang and Re 2014]. Figure 10(c) shows the Downpour [Dean et al. 2012] framework implemented in SINGA. Similar to the synchronous Sandblaster, all workers send requests to a global server group. 


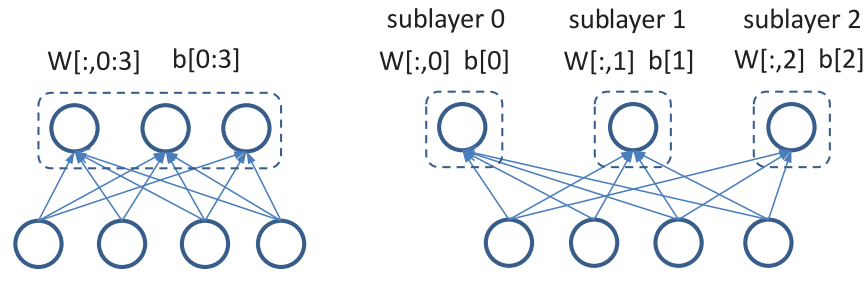

Fig. 11. Partition the hidden layer in Figure 4(a).

We divide workers into several groups, each running independently and working on parameters from the last update response. Like Sandblaster, this framework also requires the servers to have large bandwidth to handle requests for multiple worker groups. Figure 10(d) shows the distributed Hogwild framework, in which each node contains a complete server group and a complete worker group. Parameter updates are done locally so that communication cost during each training step is minimized. However, the server group must periodically synchronize with neighboring groups to improve the training convergence. The topology (connections) of server groups can be customized (the default topology is an all-to-all connection). This framework is most widely used for a single-node multi-GPU environment, where server groups synchronize via shared memory. For a large cluster, the synchronization among server groups would incur significant overhead and delay.

Asynchronous training can improve the convergence rate to some degree. But the improvement typically diminishes when there are more model replicas, as the delay (or staleness) of parameter updates would increase. A more scalable training framework should combine both synchronous and asynchronous training. In SINGA, users can run a hybrid training framework by launching multiple worker groups that run asynchronously to improve the convergence rate. Within each worker group, multiple workers run synchronously to accelerate one training iteration. Given a fixed budget (e.g., number of nodes in a cluster), there are opportunities to find one optimal hybrid training framework that trades off between the convergence rate and efficiency to achieve the minimal training time.

\subsection{Neural Network Partitioning}

In this section, we describe how SINGA partitions the neural net to support data parallelism, model parallelism, and hybrid parallelism within one worker group.

SINGA partitions a neural net at the granularity of a layer. Every layer's feature blob is considered a matrix whose rows are feature vectors. Thus, the layer can be split on two dimensions. Partitioning on dimension 0 (also called the batch dimension) slices the feature matrix by row. For instance, if the mini-batch size is 256 and the layer is partitioned into two sublayers, each sublayer would have 128 feature vectors in its feature blob. Partitioning on this dimension has no effect on the parameters, as every Param object is replicated in the sublayers. Partitioning on dimension 1 (also called the feature dimension) slices the feature matrix by column. For example, suppose that the original feature vector has 50 units; after partitioning into two sublayers, each sublayer would have 25 units. This partitioning splits Param objects, as shown in Figure 11. Both the bias vector and weight matrix are partitioned into two sublayers (workers).

Network partitioning is conducted while creating the NeuralNet instance. SINGA extends a layer into multiple sublayers. Each sublayer is assigned a location ID, based on which it is dispatched to the corresponding worker. Advanced users can also directly specify the location ID for each layer to control the placement of layers onto workers. For the MDNN model in Figure 6, users can configure the layers in the image path with 
location ID 0 and the layers in the text path with location ID 1, making the two paths run in parallel. Similarly, for the Char-RNN model shown in Figure 8, we can place the layers of different colors onto different workers. Connection layers will be automatically added to connect the sublayers. For instance, if two connected sublayers are located at two different workers, then a pair of bridge layers is inserted to transfer the feature (and gradient) blob between them. When two layers are partitioned on different dimensions, a concatenation layer that concatenates feature rows (or columns) and a slice layer that slices feature rows (or columns) are inserted. Connection layers help make the network communication and synchronization transparent to the users.

When every worker computes the gradients of the entire model parameters, we refer to this process as data parallelism. When different workers compute the gradients of different parameters, we refer to this process model parallelism. In particular, partitioning on dimension 0 of each layer results in data parallelism, whereas partitioning on dimension 1 results in model parallelism. Moreover, SINGA supports hybrid parallelism wherein some workers compute the gradients of the same subset of model parameters, whereas other workers compute on different model parameters. For example, to implement the hybrid parallelism in Krizhevsky [2014] for the CNN model, we set partition_dim $=0$ for lower layers and partition_dim $=1$ for higher layers. The following list summarizes the partitioning strategies, and their trade-off is analyzed in Section 5.4:

(1) Partitioning all layers into different subsets $\rightarrow$ model parallelism.

(2) Partitioning each single layer into sublayers on batch dimension $\rightarrow$ data parallelism.

(3) Partitioning each single layer into sublayers on feature dimension $\rightarrow$ model parallelism.

(4) Hybrid partitioning of strategies 1,2 , and $3 \rightarrow$ hybrid parallelism.

\subsection{Optimization}

Distributed training (i.e, partitioning the neural net and running workers over different layer partitions) increases the computation power (i.e., FLOPS). However, it introduces overhead in terms of communication and synchronization. Suppose that we have a homogeneous computation environment-that is, all workers run at the same speed and get the same workload (e.g., the same number of training samples and same size of feature vectors). In this case, we can ignore the synchronization overhead and analyze only the communication cost. The communication cost is mainly attributed to the data transferred through PCIe over multiple GPUs in a single node or through the network in a cluster. To cut down the overall overhead, first we try to reduce the amount of data to be transferred. Furthermore, we try to parallelize the computation and communication to hide the communication time. Here we discuss synchronous training only (i.e., a single worker group), which has the identical theoretical convergence as training in a single worker. Optimization techniques that may affect the convergence rate of SGD are not considered, such as asynchronous SGD (i.e., multiple worker groups) and parameter compression [Seide et al. 2014]. The following analysis works for training either over multiple CPU nodes or over multiple GPU cards on a single node.

5.4.1. Reducing Data Transferring. There are two types of data transferring in distributed training. First, the feature vectors may be transferred as messages if two connected layers are located in different workers (e.g., by model parallelism). Second, the parameters (values and gradients) are transferred for aggregation if they are replicated due to data parallelism. The guideline for reducing data transferring specifies to do data parallelism for layers with fewer parameters and do model parallelism for layers with smaller feature vectors. To illustrate, we use the popular benchmark model AlexNet 


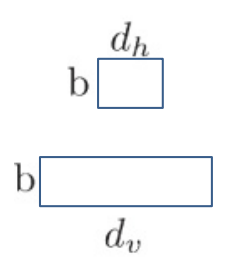

(a) Two fully connected layers

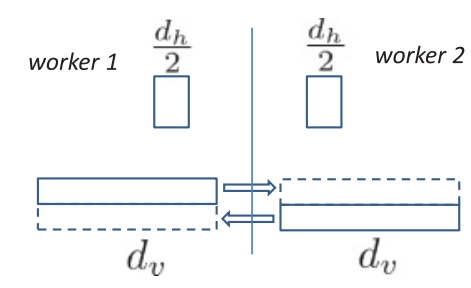

(b) Partition on hidden layer

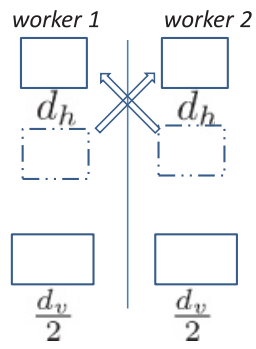

(c) Partition on visible layer

Fig. 12. Distributed computing for fully connected layers.

as an example. AlexNet is a feed-forward model with a single path; the $i^{\text {th }}$ layer depends on the $(i-1)^{t h}$ layer directly. It is not feasible to parallelize subsets of layers as in MDNN, and therefore we do not consider the first partitioning strategy. Next we discuss every type of layer involved in AlexNet, one by one.

Convolutional layers contain $5 \%$ of the total parameters but $90 \%$ to $95 \%$ of the computation, according to AlexNet [Krizhevsky 2014]. It is essential to distribute the computation from these layers. Considering that convolutional layers have large feature vectors and a small amount of parameters, it is natural to apply data parallelism.

Fully connected layers occupy $95 \%$ of the total parameters and $5 \%$ to $10 \%$ of computation [Krizhevsky 2014], and therefore we should avoid data parallelism and use model parallelism for them. Particularly, with data parallelism, the communication overhead per worker is $O(p)$, where $p$ is the size of the (replicated) parameters. Let $b$ be the effective mini-batch size (summed over all workers), let $K$ be the number of workers, and let $d_{v}$ (respectively, $d_{h}$ ) be the length of the visible (respectively, hidden) feature vector. Figure 12(b) shows the case for data partitioning for the visible layer and model partitioning for the hidden layer; the overhead is $O\left(b * d_{v}\right)$ for exchanging the visible features. Figure 12(c) applies model partitioning for the visible layer, whose overhead comes from exchanging the hidden features (i.e., $\left.O\left(b * d_{h}\right)\right)$. For the first fully connected layer in AlexNet, $p$ is about 177 million and $d_{v}=d_{h}=4,096$. In other words, $p>b * d_{v}$ and $p>b * d_{h}$, and hence data parallelism is costlier than model parallelism.

For pooling layers and local responsive normalization layers, each neuron depends on many neurons from their source layers. Moreover, they are interleaved with convolutional layers, and thus it is cheaper to apply data parallelism than model parallelism for them. For the remaining layers, they do not have parameters and their neurons depend on source neurons element-wise, and hence their partitioning strategies just need to be consistent with their source layers. Consequently, a simple hybrid partitioning strategy for AlexNet [Krizhevsky 2014] could be applying data parallelism for layers before (or under) the first fully connected layer and then applying model parallelism or no parallelism for all other layers. Currently, we require users to configure the partitioning strategy for each layer to get the preceding hybrid partitioning scheme. Automatic optimization and configuration is left as a future work. Reducing data transfer could save power but may not bring speed improvement if the communication cost is hidden due to overlapping with computation, as described in the following.

5.4.2. Overlapping Computation and Communication. Overlapping the computation and communication is another common technique for system optimization. In SINGA, the communication comprises transferring parameter gradients and values, and transferring layer data and gradients. First, for parameter gradients and values, we can send them asynchronously while computing other layers. Take Figure 4 as an example. After 


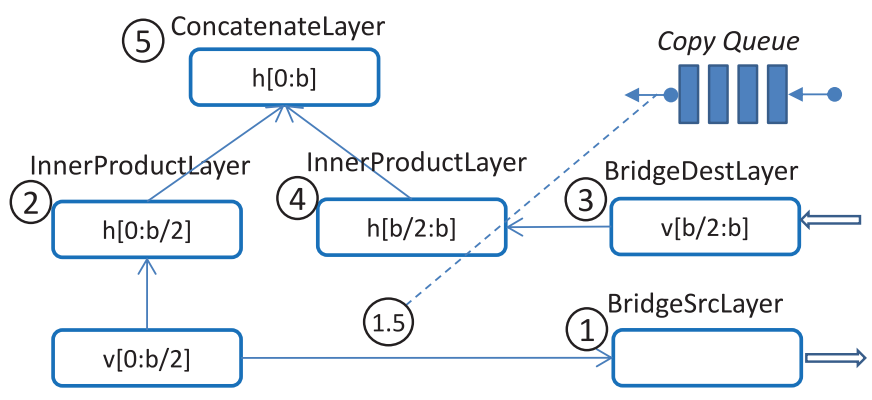

Fig. 13. Parallelize computation and communication for a GPU worker.

the hidden layer finishes ComputeFeature, we can send the gradients asynchronously to the server for updates while the worker continues to load data for the next iteration. The updated parameters are transferred back to the server by pushing a copy operation into the Copy queue as shown in Figure 13, which is checked and executed by the worker. Second, the transferring of layer data and gradients typically comes from model partitioning, as discussed in Section 5.4.1. In this case, each worker owns a small subset of data and fetches all of the rest from other workers. To overlap the computation and communication, each worker can just initiate the communication and then compute over its own data asynchronously. Take Figure 12(b) as an example. To parallelize the computation and communication, SINGA runs over the layers shown in Figure 13 in order. The BridgeSrcLayer::ComptueFeature initiates the sending operations and returns immediately. The BridgeDestLyer::ComputeFeature waits until data arrives (by checking a signal for the ending of data transfer). All layers are sorted in topology order followed by communication priority.

\section{EXPERIMENTAL STUDY}

We developed SINGA using C++ on Linux platforms. OpenBLAS and cuDNN are integrated for accelerating linear algebra and neural net operations. ZeroMQ is used for message passing. In this section, we evaluate SINGA with real-life multimedia applications. Specifically, we used SINGA to train the models discussed in Section 4.2, which required little development effort since SINGA comes with many built-in layers and algorithms. We then measured SINGA's training performance in terms of efficiency and scalability when running on CPUs and GPUs. We found that SINGA is more efficient than other open-source systems, and it is scalable for both synchronous and asynchronous training.

\subsection{Applications of SINGA}

We trained models for the example applications in Section 4.2 using SINGA. Users can train these models following the instructions online. ${ }^{5}$ The neural nets are configured using the built-in layers as shown in Figures 6, 7, and 8.

Multimodal retrieval. We trained the MDNN model for the multimodal retrieval application. We used the NUS-WIDE dataset [Chua et al. 2009], which has roughly 180,000 images after removing images without tags or from nonpopular categories. Each image is associated with several tags. We used Word2Vec [Mikolov et al. 2013] to learn a word embedding for each tag and aggregated the embedding of all tags from the same image as a text feature. Figure 14 shows sample search results. We first used images as queries to retrieve similar images and text documents. It can be seen that

\footnotetext{
$\overline{5}$ http://singa.apache.org/v0.3.0/en/docs/examples.html.
} 


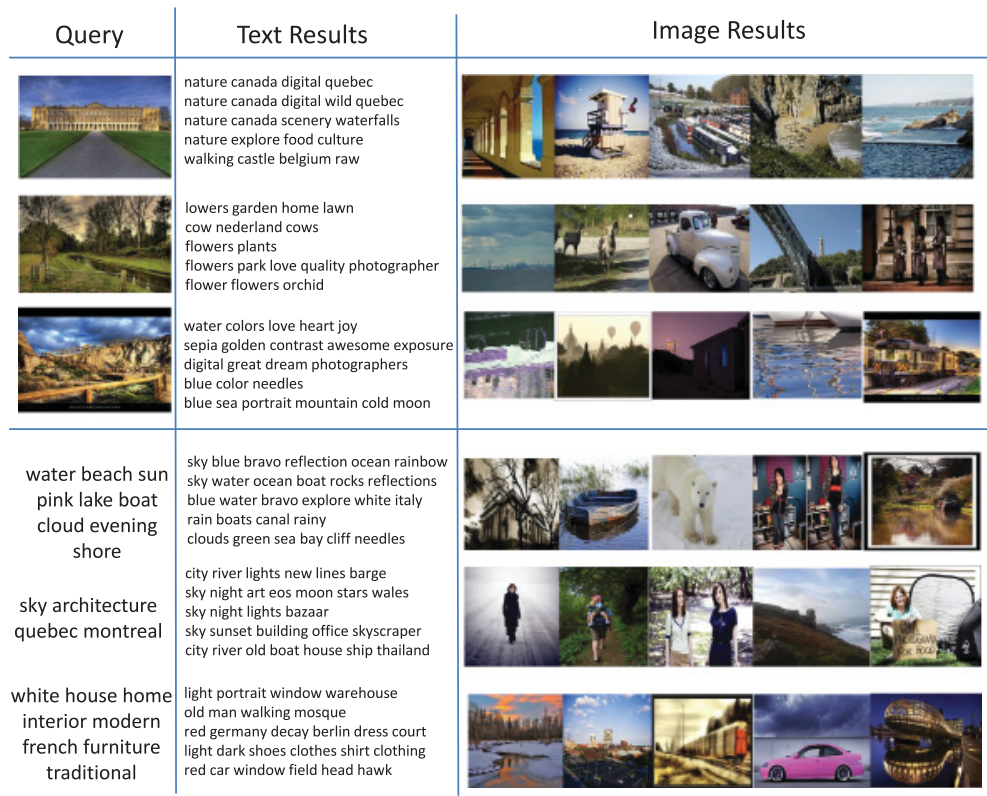

Fig. 14. Multimodal retrieval. The top five similar text documents (one line per document) and images are displayed.

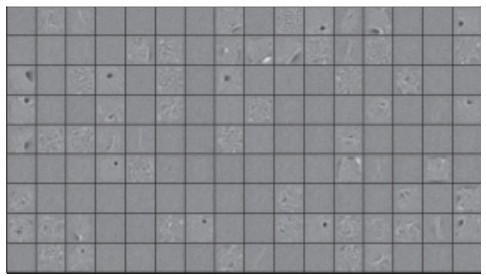

(a) Bottom RBM weight matrix

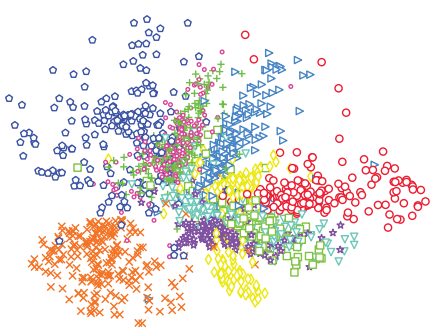

(b) Top layer features

Fig. 15. Visualization of the weight matrix in the bottom RBM and top layer features in the deep autoencoder.

image results are more relevant to the queries. For instance, the first image result of the first query is relevant because both images are about architecture, but the text results are not very relevant. This can be attributed to the large semantic gap between different modalities, making it difficult to locate semantically relevant objects in the latent (representation) space.

Dimensionality reduction. We trained RBM models to initialize the deep autoencoder for dimensionality reduction. We used the MNIST $^{6}$ dataset, consisting of 70,000 images of handwritten digits. Following the configuration used in Hinton and Salakhutdinov [2006], we set the size of each layer as $784 \rightarrow 1000 \rightarrow 500 \rightarrow 250 \rightarrow 2$. Figure 15(a) visualizes sample columns of the weight matrix of the bottom (first) RBM. We can see that Gabor-like filters are learned. Figure 15(b) depicts the features extracted from the top layer of the autoencoder, wherein one point represents one image. Different colors represent different digits. We can see that most images are well clustered according to

\footnotetext{
$\overline{{ }^{6} \mathrm{http}: / / \text { yann.lecun.com/exdb/mnist/. }}$
} 


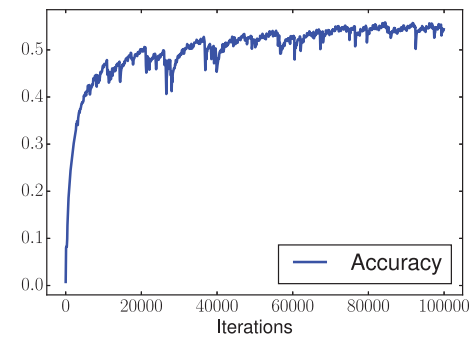

(a) Training accuracy

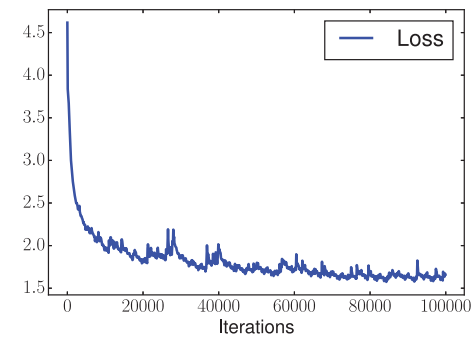

(b) Training loss

Fig. 16. Training accuracy and loss of Char-RNN.

the ground truth, except for images of digits 4 and 9 (central part), which have some overlap (in practice, handwritten 4 and 9 digits are fairly similar in shape).

Char-RNN. We used the Linux kernel source code extracted using an online script ${ }^{7}$ for this application. The dataset is about $6 \mathrm{MB}$. The RNN model is configured similar to Figure 8. Since this dataset is small, we used one stack of recurrent layers (Figure 8 has two stacks). The training loss and accuracy is shown in Figure 16. We can see that the Char-RNN model can be trained to predict the next character given previous characters in the source code more and more accurately. There are some fluctuations due to the variance of data samples in different mini-batches (the loss and accuracy are computed per mini-batch).

\subsection{Training Performance Evaluation on the CPU}

We evaluated SINGA's training efficiency and scalability for both synchronous and asynchronous frameworks on a single multicore node and on a cluster of commodity servers.

6.2.1. Methodologies. The deep convolution neural network ${ }^{8}$ for image classification was used as the training model for benchmarking. The training was conducted over the CIFAR10 dataset, ${ }^{9}$ which has 50,000 training images and 10,000 test images. For the single-node setting, we used a 24-core server with 500GB memory. The 24 cores are distributed into 4 NUMA nodes (Intel Xeon 7540). Hyperthreading is turned on. For the multinode setting, we used a 32-node cluster. Each cluster node is equipped with a quad-core Intel Xeon 3.1GHz CPU and 8GB memory. The cluster nodes are connected by a $1 \mathrm{Gbps}$ switch.

6.2.2. Synchronous Training. We compared SINGA with $\mathrm{CXXNET}^{10}$ and Caffe [Jia et al. 2014]. All three systems use OpenBlas to accelerate matrix multiplications. Both CXXNET and Caffe were compiled with their default optimization levels: O3 for the former and $\mathrm{O} 2$ for the latter. We observed that because synchronous training has the same convergence rate as that of sequential SGD, all systems would converge after same number of iterations (i.e., mini-batches). This means that the difference in total training time among these systems is attributed to the efficiency of a single iteration. Therefore, we only compared the training time for 1 iteration. We ran 100 iterations for each system and averaged the result time over 50 iterations, 30th to 80th iterations, to avoid the effect of starting and ending phases.

\footnotetext{
${ }^{7}$ http://cs.stanford.edu/people/karpathy/char-rnn.

${ }^{8} \mathrm{https}$ ://code.google.com/p/cuda-convnet/.

${ }^{9}$ https://www.cs.toronto.edu/ kriz/cifar.html.

${ }^{10} \mathrm{https}: / / \mathrm{github} . \mathrm{com} / \mathrm{dmlc} / \mathrm{cxxnet}$.
} 


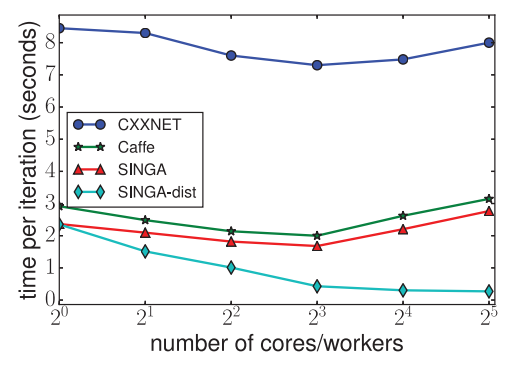

(a) On the single node

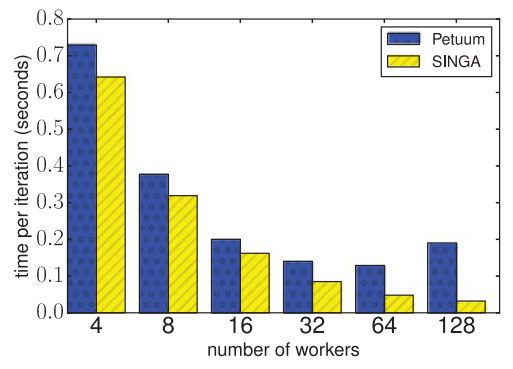

(b) On the 32-node cluster

Fig. 17. Synchronous training.

On the 24-core single node, we used 256 images per mini-batch and varied the number of OpenBlas's threads. The result is shown in Figure 17(a). SINGA-dist represents the SINGA configuration in which there are multiple workers; each worker has one OpenBlas thread. ${ }^{11}$ In contrast, SINGA represents the configuration that has only one worker. We configured SINGA-dist with the cluster topology consisting of one server group with four servers and one worker group with varying number of worker threads (Figure 17(a)). In other words, SINGA-dist ran as the in-memory Sandblaster framework. We can see that SINGA-dist has the best overall performance: it is the fastest for each number of threads, and it is also the most scalable. Other systems using multithreaded OpenBlas scale poorly. This is because OpenBlas has little awareness of the application, and hence it cannot be fully optimized. For example, it may only parallelize specific operations. such as large matrix multiplications. In contrast, SINGA-dist partitions the mini-batch equally between workers and achieves parallelism at the worker level. Another limitation of OpenBlas, as shown in Figure 17(a), is that when there were more than eight threads, the overheads caused by cross-CPU memory access [Tan et al. 2015] started to have a negative effect on overall performance.

On the 32-node cluster, we compared SINGA against another distributed machine learning framework called Petuum [Dai et al. 2013]. Petuum runs Caffe as an application to train deep learning models. It implements a parameter server to perform updates from workers (clients) while the workers run synchronously. We used a larger mini-batch size (512 images) and disabled OpenBlas multithreading. We configured SINGA's cluster topology to realize the AllReduce framework: there is 1 worker group and 1 server group, and in each node there are 4 workers and 1 server. We varied the size of worker group from 4 to 128 and the server group size from 1 to 32 . We note that one drawback of synchronous distributed training is that it cannot scale to too many nodes because there is typically an upper limit on the mini-batch size (i.e., 1,024 images). Consequently, there is an upper bound on the number of workers we can launch (i.e., 1,024 workers); otherwise, some workers will not be assigned any image to train. Figure 17(b) shows that SINGA achieves almost linear scalability. In contrast, Petuum scales up to 64 workers but becomes slower when 128 workers are launched. This might be attributed to the communication overheads at the parameter server and the synchronization delays among workers.

6.2.3. Asynchronous Training. We compared SINGA against Caffe, which has support for in-memory asynchronous training. On the single node, we configured Caffe to use the in-memory Hogwild [Recht et al. 2011] framework and SINGA to use the in-memory

\footnotetext{
${ }^{11}$ OPENBLAS_NUM_THREADS=1.
} 


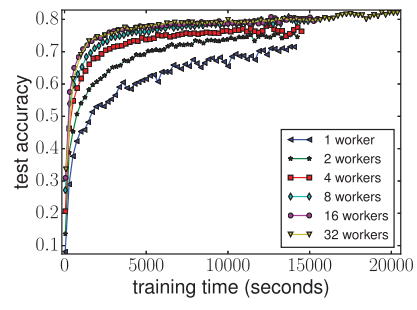

(a) Caffe on the single node

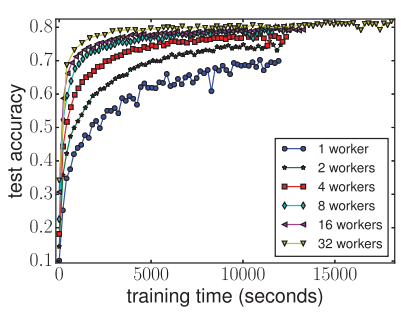

(b) SINGA on the single node

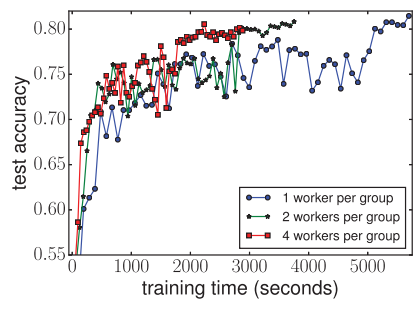

(c) SINGA on the cluster

Fig. 18. Asynchronous training.

Downpour framework. Their main difference is that parameter updates are done by workers in Caffe and by a single server (thread) in SINGA. Figure 18(a) and (b) show the model accuracy versus training time with varying numbers of worker groups (i.e., model replicas). Every worker processed 16 images per iteration for a total of 60,000 iterations. We can see that SINGA trains faster than Caffe. Both systems scale well as the number of workers increases, both in terms of the time to reach the same accuracy and of the final converged accuracy. We can also observe that the training takes longer with more workers. This is due to the increased overhead in context switching when there are more threads (workers). Finally, we note from the results that the performance difference becomes smaller when the cluster size (i.e., the number of model replicas) reaches 16 . This implies that there would be little benefit in having too many model replicas. Thus, we fixed the number of model replicas (i.e., worker groups) to 32 in the following experiments for the distributed asynchronous training.

On the 32-node cluster, we used a mini-batch of 16 images per worker group and 60,000 training iterations. We varied the number of workers within one group and configured the distributed Downpour framework to have 32 worker groups and 32 servers per server group (one server thread per node). We can see from Figure 18(c) that with more workers, the training is faster because each worker processes fewer images. However, the training is not as stable as in the single-node setting. This may be caused by the delay (staleness) of parameter synchronization between workers, which is not present in single-node training because parameter updates are immediately visible on the shared memory. The final stage of training (i.e., last few points of each line) is stable because there is only one worker group running during that time, namely the testing group. We note that using a warm-up stage, which trains the model using a single worker group at the beginning, may help to stabilize the training as reported in Google's DistBelief system [Dean et al. 2012].

\subsection{Training Performance Evaluation on the GPU}

We evaluated the training performance of SINGA running on GPUs. We first analyzed the two optimization techniques discussed in Section 5.4, then we compared SINGA with other open-source, state-of-the-art systems.

6.3.1. Methodologies. We used the online benchmark model from Soumith ${ }^{12}$ as the training workload. The model is adapted from the AlexNet model [Krizhevsky 2014] with some layers omitted. Two sets of hardware are used in our experiments, whose specs and software configurations are shown in Table III.

6.3.2. Overlapping Communication and Computation. In Section 5.4.2, we analyzed the optimization technique for hiding the communication overhead by overlapping it with

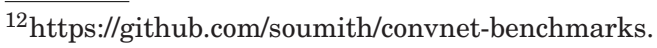


Table III. Specs of Hardware and Software

\begin{tabular}{|l|c|c|c|c|c|}
\hline Type & CPU & Memory & GPU & CUDA & cuDNN \\
\hline Single node & Intel i7-5820K & 16GB & GTX 970 (4GB) & 7.0 & 4.0 \\
\hline GPU cluster (4 nodes) & Intel i7-5820K & $64 \mathrm{~GB}$ & GTX TITAN-X (12GB) & 7.5 & 4.0 \\
\hline
\end{tabular}

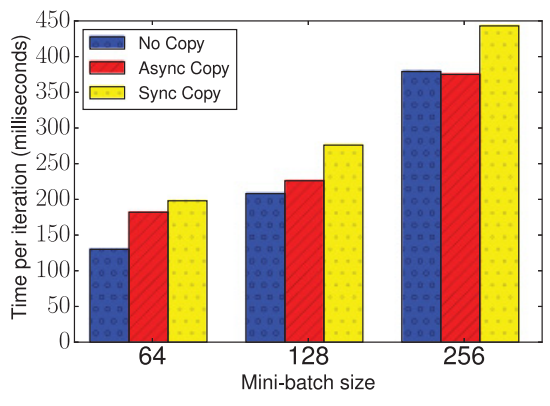

(a) Hide communication cost

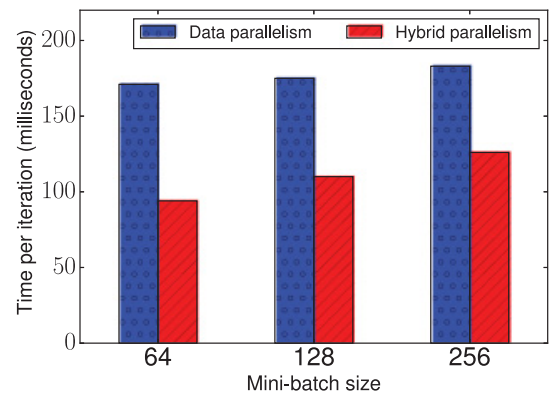

(b) Reduce data transferring

Fig. 19. Effect of optimization techniques.

the computation. Here we evaluate the effect of this technique using the single node. Particularly, we compare the efficiency in terms of time per iteration for three versions of SINGA. The No Copy version indicates that there is no communication between the GPU and CPU, which is widely used for training with a single GPU, where all operations including a parameter update are conducted on the single GPU. The other two versions conduct the BP algorithm on a GPU and parameter updating on a CPU, differing only by whether data transferring is done synchronously or asynchronously.

Figure 19(a) shows the time per iteration with different mini-batch sizes. First, we can see that No Copy is the fastest one because it has no communication cost at all. Second, Async Copy is faster than Sync Copy, which suggests that the asynchronous data transferring benefits from the overlapping communication and computation. Moreover, we can see that when the mini-batch increases, the difference between Async Copy and Sync Copy decreases. This is because for large mini-batches, the BP algorithm spends more time doing computation, which increases the overlap area of computation and communication, effectively reducing the overhead. For mini-batch size $=256$, Async Copy is even faster than No Copy, and this is because Async Copy does not do a parameter update, which is done by the server in parallel with BP. However, No Copy has to do BP and parameter updating sequentially.

6.3.3. Reducing Data Transferring. In Section 5.4.1, we discussed how hybrid partitioning is better than other strategies in terms of the overheads in transferring feature vectors between layers in different workers. To demonstrate its effectiveness, we ran SINGA on the single node using two partitioning strategies, namely data partitioning and hybrid partitioning, for the first fully connected layer in AlexNet. Two workers are launched (one per GPU). Figure 19(b) shows the time per iteration with different mini-batch sizes. We can see that hybrid partitioning has better performance than data partitioning. For data partitioning, only parameter gradients and values are transferred, which is independent of the mini-batch size, and thus the time per iteration does not change much when the mini-batch size increases. For hybrid partitioning, when the mini-batch size increases, more feature vectors would be transferred, and then the time increases.

6.3.4. Comparison With Other Systems. We also compared SINGA to four other stateof-the-art deep learning systems: Caffe [Jia et al. 2014], MxNet [Chen et al. 2015], 


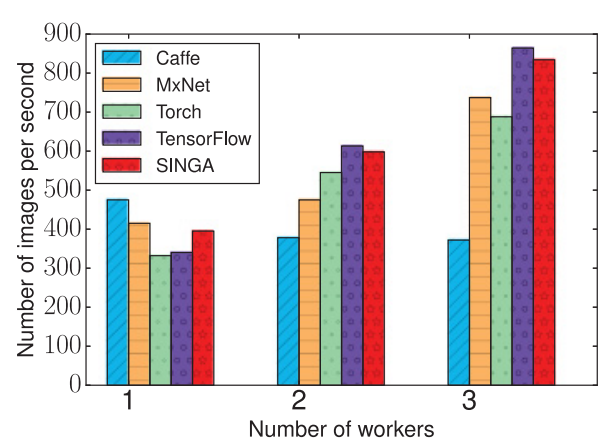

(a) A single node with multi-GPUs

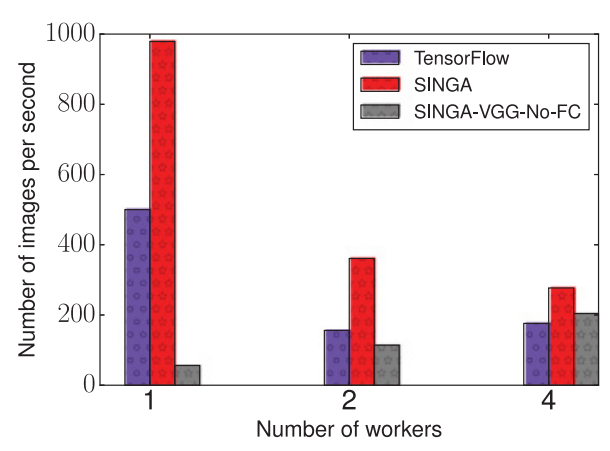

(b) A GPU cluster with four nodes

Fig. 20. Performance comparison of open-source systems.

Torch7 [Collobert et al. 2011], and TensorFlow [Abadi et al. 2015]. The performance is measured using the throughput (i.e., number of processed images per second). We used (or adapted) the scripts (or instructions) from each system's multi-GPU examples. ${ }^{13,14,15,16}$ We simply adapted the online code for the experiments, and better performance could be achieved with further tuning.

We first compared the throughput of training on a single node with different numbers of workers (GPUs). We varied the number of workers from one to three, where each worker ran on a GPU. Because TensorFlow ran out of memory with batch size $=128$ (the setting used by Soumith's benchmark), we decreased the batch size to 96 for all runnings. The results are shown in Figure 20(a).

Caffe has the best single-worker performance. This is because there is no parameter transferring between the GPU and CPU, whereas others have parameters transferring. For example, SINGA has to transfer the parameter from the worker (on the GPU) to the server (on the CPU). However, its performance decreases when more workers are added. This is because (1) the parameters have to be transferred among GPUs (via CPUs), which brings in communication cost, and (2) its tree reduction communication pattern (see the link in the footnote 13) incurs more cost than the all-to-one or allreduce communication pattern used by other systems when there are more than two workers.

For other systems, they have similar performance for the single-worker case, as they all use the same cuDNN library for most of the computation. Note that their throughput is lower than Soumith's benchmark because the GPUs are slower than Soumith's and there is communication cost, whereas Soumith's benchmark does not involve parameter transferg. Thanks to the optimization techniques introduced in Section 5.4, SINGA has almost linear scalability. TensorFlow shows the best scalability among all tested systems.

Next we ran SINGA and TensorFlow in the GPU cluster using the synchronous training framework. We varied the number of nodes (one GPU worker per node), as shown in Figure 20(b). For TensorFlow, we launched one parameter server, which communicates with all workers via gRPC. For SINGA, we created a single server group

\footnotetext{
${ }^{13}$ Caffe: https://github.com/BVLC/caffe/blob/master/docs/multigpu.md.

${ }^{14} \mathrm{MxNet:}$ https://mxnet.readthedocs.io/en/latest/how_to/multi_devices.html.

${ }^{15}$ Torch7: https://github.com/soumith/imagenet-multiGPU.torch.

${ }^{16}$ TensorFlow: https://www.tensorflow.org/versions/r0.8/tutorials/deep_cnn/index.html and https://github. com/tensorflow/models/tree/master/inception.
} 
with one server, which is in the same process as the first worker and communicates with other workers using ZeroMQ. We reduced the size of the first fully connected layer to 128, as this layer has a big parameter matrix whose size exceeds the limit of the Protobuf message used by TensorFlow. Consequently, the performance for a single node (i.e., single GPU) is better than that in Figure 20(a). We can see that SINGA performs much better than TensorFlow in terms of throughput. It is likely caused by the network communication, which is not well optimized in TensorFlow. SINGA avoids some communication cost by running the first worker and the parameter server in the same process (they transfer messages via sharing memory). Both systems show poor scalability when there are more than two workers (nodes). On the one hand, nodeto-node communication cost and synchronization cost are introduced when there are more than two workers. One the other hand, the AlexNet model has too many parameters, which makes the communication the bottleneck. To verify this explanation, we conducted another set of experiments using the VGG model [Simonyan and Zisserman 2014] with fully connected layers omitted, denoted as VGG-No-FC. This model has 10 convolutional layers and a small amount of parameters, which makes it more computation intensive than the AlexNet model. The result in Figure 20(b) shows that SINGA has good scalability for this model, which confirms our explanation. To conclude, distributed training is more suitable for models that are computation intensive and have a small amount of parameters.

\section{CONCLUSION}

In this article, we proposed SINGA, a distributed deep learning platform for supporting multimedia applications. SINGA offers a simple and intuitive programming model, making it accessible even to nonexperts. SINGA is extensible and able to support a wide range of multimedia applications requiring different deep learning models. The flexible training architecture gives the user the chance to balance the trade-off between the training efficiency and convergence rate. Optimization techniques are applied to improve the training performance. We demonstrated the use of SINGA for representative multimedia applications using a CPU cluster and a single node with multiple GPU cards, and have shown that the platform is both usable and scalable.

\section{ACKNOWLEDGMENTS}

We would like to thank the SINGA team members and NetEase for their contributions to Apache SINGA, the anonymous reviewers for their insightful and constructive comments, and the NUS SeSaMe Center for sharing the GPU cluster for our experiments.

\section{REFERENCES}

Martín Abadi, Ashish Agarwal, Paul Barham, Eugene Brevdo, Zhifeng Chen, Craig Citro, Greg S. Corrado et al. 2015. TensorFlow: Large-scale machine learning on heterogeneous systems. arXiv:1603.04467. http://tensorflow.org/.

Frédéric Bastien, Pascal Lamblin, Razvan Pascanu, James Bergstra, Ian J. Goodfellow, Arnaud Bergeron, Nicolas Bouchard, and Yoshua Bengio. 2012. Theano: New features and speed improvements. In Proceedings of the Deep Learning Workshop (NIPS'12).

Tianqi Chen, Bing Xu, Chiyuan Zhang, and Carlos Guestrin. 2016b. Training deep nets with sublinear memory cost. arXiv:1604.06174. http://arxiv.org/abs/1604.06174

Tianqi Chen, Mu Li, Yutian Li, Min Lin, Naiyan Wang, Minjie Wang, Tianjun Xiao, Bing Xu, Chiyuan Zhang, and Zheng Zhang. 2015. MXNet: A flexible and efficient machine learning library for heterogeneous distributed systems. arXiv:1512.01274.

Jianmin Chen, Rajat Monga, Samy Bengio, and Rafal Józefowicz. 2016a. Revisiting distributed synchronous SGD. arXiv:1604.00981. http://arxiv.org/abs/1604.00981

Trishul Chilimbi, Yutaka Suzue, Johnson Apacible, and Karthik Kalyanaraman. 2014. Project Adam: Building an efficient and scalable deep learning training system. In Proceedings of the 11th USENIX 
Conference on Operating Systems Design and Implementation (OSDI'14). 571-582. https://www.usenix. org/conference/osdi14/technical-sessions/presentation/chilimbi.

Tat-Seng Chua, Jinhui Tang, Richang Hong, Haojie Li, Zhiping Luo, and Yan-Tao. Zheng. 2009. NUS-WIDE: A real-world Web image database from National University of Singapore. In Proceedings of the ACM International Conference on Image and Video Retrieval (CIVR'09). Article No. 48.

Dan Claudiu Ciresan, Ueli Meier, Luca Maria Gambardella, and Jürgen Schmidhuber. 2010. Deep big simple neural nets excel on handwritten digit recognition. arXiv:1003.0358.

Adam Coates, Brody Huval, Tao Wang, David J. Wu, Bryan C. Catanzaro, and Andrew Y. Ng. 2013. Deep learning with COTS HPC systems. In Proceedings of the 30th International Conference on Machine Learning (ICML'13). 1337-1345.

R. Collobert, K. Kavukcuoglu, and C. Farabet. 2011. Torch7: A Matlab-like environment for machine learning. In Proceedings of the BigLearn Workshop (NIPS'11).

Wei Dai, Jinliang Wei, Xun Zheng, Jin Kyu Kim, Seunghak Lee, Junming Yin, Qirong Ho, and Eric P. Xing. 2013. Petuum: A framework for iterative-convergent distributed ML. arXiv:1312.7651. http://arxiv.org/abs/1312.7651

Jeffrey Dean, Greg Corrado, Rajat Monga, Kai Chen, Matthieu Devin, Quoc V. Le, Mark Z. Mao, Marc'Aurelio Ranzato, Andrew W. Senior, Paul A. Tucker, Ke Yang, and Andrew Y. Ng. 2012. Large scale distributed deep networks. In Advances in Neural Information Processing Systems (NIPS'12). 1232-1240.

John C. Duchi, Elad Hazan, and Yoram Singer. 2011. Adaptive subgradient methods for online learning and stochastic optimization. Journal of Machine Learning Research 12, 2121-2159. http://dl.acm.org/ citation.cfm?id=2021068

Fangxiang Feng, Xiaojie Wang, and Ruifan Li. 2014. Cross-modal retrieval with correspondence autoencoder. In Proceedings of the 22nd ACM International Conference on Multimedia (MM'14). 7-16. DOI : http://dx.doi.org/10.1145/2647868.2654902

Kaiming He, Xiangyu Zhang, Shaoqing Ren, and Jian Sun. 2015. Deep residual learning for image recognition. arXiv:1512.03385.

Geoffrey Hinton and Ruslan Salakhutdinov. 2006. Reducing the dimensionality of data with neural networks. Science 313, 5786, 504-507.

Yangqing Jia, Evan Shelhamer, Jeff Donahue, Sergey Karayev, Jonathan Long, Ross Girshick, Sergio Guadarrama, and Trevor Darrell. 2014. Caffe: Convolutional architecture for fast feature embedding. arXiv:1408.5093.

Dawei Jiang, Gang Chen, Beng Chin Ooi, Kian-Lee Tan, and Sai Wu. 2014. epiC: An extensible and scalable system for processing big data. Proceedings of the VLDB Endowment 7, 7, 541-552. http://www.vldb.org/ pvldb/vol7/p541-jiang.pdf.

Alex Krizhevsky. 2014. One weird trick for parallelizing convolutional neural networks. arXiv:1404.5997.

Alex Krizhevsky, Ilya Sutskever, and Geoffrey E. Hinton. 2012. ImageNet classification with deep convolutional neural networks. In Advances in Neural Information Processing Systems 25 (NIPS'12). 1106-1114.

Quoc V. Le, Marc'Aurelio Ranzato, Rajat Monga, Matthieu Devin, Greg Corrado, Kai Chen, Jeffrey Dean, and Andrew Y. Ng. 2012. Building high-level features using large scale unsupervised learning. In Proceedings of the International Conference on Machine Learning (ICML'12).

Yann LeCun, Léon Bottou, Genevieve B. Orr, and Klaus-Robert Müller. 1996. Efficient BackProp. In Neural Networks: Tricks of the Trade. Springer, 9-50. DOI : http://dx.doi.org/10.1007/3-540-49430-8_2

Mu Li, David G. Andersen, Jun Woo Park, Alexander J. Smola, Amr Ahmed, Vanja Josifovski, James Long, Eugene J. Shekita, and Bor-Yiing Su. 2014. Scaling distributed machine learning with the parameter server. In Proceedings of the 11th USENIX Symposium on Operating Systems Design and Implementation (OSDI'14). 583-598. https://www.usenix.org/conference/osdi14/technical-sessions/presentation/li_mu.

Tomas Mikolov, Ilya Sutskever, Kai Chen, Gregory S. Corrado, and Jeffrey Dean. 2013. Distributed representations of words and phrases and their compositionality. In Advances in Neural Information Processing Systems (NIPS'13). 3111-3119.

Tomas Mikolov, Stefan Kombrink, Lukás Burget, Jan Cernocký, and Sanjeev Khudanpur. 2011. Extensions of recurrent neural network language model. In Proceedings of the 2011 IEEE International Conference on Acoustics, Speech, and Signal Processing (ICASSP'11). IEEE, Los Alamitos, CA, 5528-5531. DOI : http://dx.doi.org/10.1109/ICASSP.2011.5947611

Beng Chin Ooi, Kian-Lee Tan, Sheng Wang, Wei Wang, Qingchao Cai, Gang Chen, Jinyang Gao et al. 2015. SINGA: A distributed deep learning platform. In Proceedings of the ACM Multimedia Conference.

Thomas Paine, Hailin Jin, Jianchao Yang, Zhe Lin, and Thomas S. Huang. 2013. GPU asynchronous stochastic gradient descent to speed up neural network training. arXiv:1312.6186. 
Benjamin Recht, Christopher Re, Stephen J. Wright, and Feng Niu. 2011. Hogwild: A lock-free approach to parallelizing stochastic gradient descent. In Advances in Neural Information Processing Systems (NIPS'11). 693-701.

Frank Seide, Hao Fu, Jasha Droppo, Gang Li, and Dong Yu. 2014. 1-bit stochastic gradient descent and its application to data-parallel distributed training of speech DNNs. In Proceedings of the 15th Annual Conference of the International Speech Communication Association (INTERSPEECH'14). 1058-1062.

Karen Simonyan and Andrew Zisserman. 2014. Very deep convolutional networks for large-scale image recognition. arXiv:1409.1556. http://arxiv.org/abs/1409.1556

Christian Szegedy, Wei Liu, Yangqing Jia, Pierre Sermanet, Scott Reed, Dragomir Anguelov, Dumitru Erhan, Vincent Vanhoucke, and Andrew Rabinovich. 2014. Going deeper with convolutions. arXiv:1409.4842.

Heng Tao Shen, Beng Chin Ooi, and Kian-Lee Tan. 2000. Giving meanings to WWW images. In Proceedings of the ACM Multimedia Conference. 39-47.

Kian-Lee Tan, Qingchao Cai, Beng Chin Ooi, Weng-Fai Wong, Chang Yao, and Hao Zhang. 2015. In-memory databases: Challenges and opportunities from software and hardware perspectives. ACM SIGMOD Record 44, 2, 35-40.

Ji Wan, Dayong Wang, Steven Chu Hong Hoi, Pengcheng Wu, Jianke Zhu, Yongdong Zhang, and Jintao Li. 2014. Deep learning for content-based image retrieval: A comprehensive study. In Proceedings of the ACM Multimedia Conference. 157-166.

Xinxi Wang and Ye Wang. 2014. Improving content-based and hybrid music recommendation using deep learning. In Proceedings of the ACM Multimedia Conference. 627-636. DOI:http://dx.doi.org/10.1145/ 2647868.2654940

Wei Wang, Beng Chin Ooi, Xiaoyan Yang, Dongxiang Zhang, and Yueting Zhuang. 2014. Effective multimodal retrieval based on stacked auto-encoders. Proceedings of the VLDB Endowment 7, 8, 649-660.

Wei Wang, Gang Chen, Tien Tuan Anh Dinh, Jinyang Gao, Beng Chin Ooi, Kian-Lee Tan, and Sheng Wang. 2015a. SINGA: Putting deep learning in the hands of multimedia users. In Proceedings of the ACM Multimedia Conference.

Wei Wang, Xiaoyan Yang, Beng Chin Ooi, Dongxiang Zhang, and Yueting Zhuang. 2015b. Effective deep learning-based multi-modal retrieval. VLDB Journal 25, 1, 79-101. DOI:http://dx.doi.org/10.1007/ s00778-015-0391-4

Ren Wu, Shengen Yan, Yi Shan, Qingqing Dang, and Gang Sun. 2015. Deep Image: Scaling up image recognition. arXiv:1501.02876. http://arxiv.org/abs/1501.02876

Zuxuan Wu, Yu-Gang Jiang, Jun Wang, Jian Pu, and Xiangyang Xue. 2014. Exploring inter-feature and inter-class relationships with deep neural networks for video classification. In Proceedings of the ACM Multimedia Conference. 167-176.

Omry Yadan, Keith Adams, Yaniv Taigman, and Marc'Aurelio Ranzato. 2013. Multi-GPU training of ConvNets. arXiv:1312.5853.

Quanzeng You, Jiebo Luo, Hailin Jin, and Jianchao Yang. 2015. Joint visual-textual sentiment analysis with deep neural networks. In Proceedings of the ACM Multimedia Conference. 1071-1074. DOI : http:// dx.doi.org/10.1145/2733373.2806284.

Dong Yu, Adam Eversole, Mike Seltzer, Kaisheng Yao, Oleksii Kuchaiev, Yu Zhang, Frank Seide et al. 2014. An Introduction to Computational Networks and the Computational Network Toolkit. Microsoft Technical Report MSR-TR-2014-112. Microsoft Research.

Ce Zhang and Christopher Re. 2014. DimmWitted: A study of main-memory statistical analytics. Proceedings of the VLDB Endowment 7, 12, 1283-1294. http://www.vldb.org/pvldb/vol7/p1283-zhang.pdf.

Hanwang Zhang, Yang Yang, Huan-Bo Luan, Shuicheng Yang, and Tat-Seng Chua. 2014. Start from scratch: Towards automatically identifying, modeling, and naming visual attributes. In Proceedings of the ACM Multimedia Conference. 187-196.

Received February 2016; revised June 2016; accepted August 2016 